\title{
Satisfacción de las necesidades psicológicas básicas en las clases de Educación Física y su relación con el miedo al fallo según el género y la práctica físico-deportiva extraescolar de los estudiantes de Educación Secundaria
}

\author{
Satisfaction of the basic psychological needs in Physical Education classes and their \\ relationship with the fear of failure according to gender and the extracurricular sports \\ practice of Secondary Education students
}

\author{
Manuel Gómez-López a , Alberto Martínez Hernández, \\ Antonio Granero-Gallegos ${ }^{b}$ \\ ${ }^{a}$ Dpto. de Actividad Física y Deporte. Universidad de Murcia, España. \\ mgomezlop@um.es \\ ${ }^{b}$ Dpto. de Educación. Universidad de Almería, España.
}

\begin{abstract}
RESUMEN
El objetivo del estudio fue analizar la satisfacción de las necesidades psicológicas básicas de los estudiantes y su relación con las causas aversivas del miedo al fallo en las clases de educación física y comprobar las diferencias en función del género y de su práctica extraescolar. Para ello los participantes respondieron a la Escala de las Necesidades Psicológicas Básicas en el Ejercicio (BPNES) y el Inventario de Evaluación del Error en el Rendimiento (PFAI). La muestra estuvo compuesta por 464 alumnos de edades comprendidas entre 12 y 17 años pertenecientes a diferentes centros educativos de Educación Secundaria Obligatoria de la Región de Murcia. Los resultados reflejaron que la necesidad psicológica básica más satisfecha fue la relación con los demás, mientras que la causa aversiva más enunciada fue la de experimentar vergüenza. Se comprobó cómo los chicos se sintieron más competentes que las chicas, mientras que no se encontraron diferencias en cuanto a la percepción del miedo al fallo. Asimismo, los alumnos que practicaron actividades extraescolares mostraron los valores más altos en la satisfacción de las tres necesidades psicológicas básicas, y tener mayor miedo al fallo a causa de un futuro incierto. Finalmente, la percepción de autonomía se relacionó con un menor miedo a perder el interés de otros, mientras que la percepción de competencia y la relación con los demás lo hizo con todas las causas aversivas del miedo al fallo.
\end{abstract}

Palabras claves: Autonomía, competencia, error, vergüenza, relación con los demás.

\section{ABSTRACT}

The aim of this study is to analyze the satisfaction of the basic psychological needs of students and their relation with aversive causes which are induced by the fear of failure in physical education classes and to notice the differences depending on sex and extracurricular practice. For achieving these aims, the subjects answer to Basic Psychological Needs in Exercise Scale (BPNES) and Performance Failure Appraisal Inventory (PFAI). The sample is composed of 464 students between 12-17 years old from different schools of compulsory secondary education in Murcia. Results reflect that the basic psychological needs most fulfilled is the relatedness with other students, whereas the most aversive cause is to feel shame. It is ascertained how children feel more competent than girls, whereas there was no difference between the fear of failure. In addition, children who practice any physical practice show higher results in the three psychological needs and feel fear due to their uncertain 
Estudios Pedagógicos XLVII N 1: 303-322, 2021

SATISFACCIÓN DE LAS NECESIDADES PSICOLÓGICAS BÁSICAS EN LAS CLASES DE EDUCACIÓN FÍSICA Y SU RELACIÓN CON EL MIEDO AL FALLO SEGÚN EL GÉNERO Y LA PRÁCTICA FÍSICO-DEPORTIVA EXTRAESCOLAR ...

future. Eventually, the perception of autonomy is associated with a less fear of losing interest of others, whereas competence perception and the relatedness with others are associated with all aversive causes of fear of failure.

Key words: Autonomy, competence, failure, shame, relatedness.

\section{INTRODUCCIÓN}

La asignatura de Educación Física (EF) tiene como singularidad, que la diferencian del resto de contextos donde también se realiza actividad física, su carácter de obligatoriedad (GómezLópez, Granero-Gallegos, Baena-Extremera y Abraldes, 2014; Gómez-López, GraneroGallegos, Baena-Extremera, Bracho y Pérez, 2015; Menéndez Santurio y Fernández-Río, 2018). En este contexto de logro, surgen una serie de Necesidades Psicológicas Básicas (NPB) que cuando los estudiantes las satisfacen incrementan su compromiso, rendimiento y aprendizaje (Deci y Ryan, 1985). Deci y Ryan (2000) entienden las NPB como los nutrimentos psicológicos innatos que son esenciales para un prolongado crecimiento psicológico, integridad y bienestar. La teoría principal que fundamenta las NPB es la teoría de la autodeterminación (TAD; Ryan, Kuhl, y Deci, 1997). Esta teoría se entiende como un continuo en el que se establecen diferentes niveles de autodeterminación. Es decir, de mayor a menor grado de autodeterminación, la conducta del deportista puede estar intrínsecamente motivada, extrínsecamente motivada o a-motivada. El mayor grado de autodeterminación lo posee el deportista cuando se encuentra intrínsecamente motivado, lo que conlleva un compromiso con la práctica deportiva gracias al placer y al disfrute que obtiene con su práctica, convirtiéndose ésta en un fin en sí misma (Deci y Ryan, 1985, 2000).

Según Deci y Ryan (1991) existen tres NPB que el alumno debe satisfacer; la primera de ellas es la autonomía, la cual se refiere a la sensación que tiene el alumno de ser protagonista y partícipe de aquello que realiza, pudiendo así valorar su propia actuación y tomar decisiones a la hora de solucionar las tareas (Black y Deci, 2000). Esta autonomía mejora en el alumno cuando este siente que se valoran sus opiniones, se respetan, se tienen en cuenta sus sentimientos y se les da la oportunidad de tomar sus propias decisiones (Ryan y Deci, 2000).

Se han realizado numerosas investigaciones en las que se relaciona el apoyo a la autonomía con dos aspectos propios de la práctica deportiva, como la motivación y el estilo de vida (Aguado-Gómez, Díaz-Cueto, Hernández-Álvarez y López-Rodríguez, 2016). En relación al aspecto motivacional, se ha demostrado que es muy importante para el alumno el apoyo a la autonomía, ya que tiene una influencia positiva para la motivación intrínseca (Cox y Williams, 2008; Goudas, Biddle y Fox, 1994; Moreno, Zomeño, Marín, Ruiz y Cervelló, 2013; Standage, Duda y Ntoumanis, 2005) lo que provocará que el alumno se implique más en su aprendizaje consiguiendo de esta forma una mayor adherencia al deporte (Harris, Kuramoto, Schulzer y Retallack, 2009). Por otro lado, este apoyo a la autonomía también se relaciona positivamente con un estilo de vida activo, en el que el alumno realiza ejercicio físico de manera regular (Chatzisarantis y Hagger, 2009; Lim y Wang, 2009; Zhang, Solmon y Gu, 2012) lo que le permitirá conseguir una serie de beneficios para su salud física, mental y social (Harris et al., 2009; Monteiro et al., 2019). Siguiendo a Chengfu, Xian, y Zhang (2015), el apoyo a la autonomía que los profesores fomentan entre los alumnos favorece la satisfacción de las otras dos NPB de relacionarse con los demás (profesores, compañeros, etc.) y de competencia en las tareas encomendadas. 
SATISFACCIÓN DE LAS NECESIDADES PSICOLÓGICAS BÁSICAS EN LAS CLASES DE EDUCACIÓN FÍSICA Y SU RELACIÓN CON EL MIEDO AL FALLO SEGÚN EL GÉNERO Y LA PRÁCTICA FÍSICO-DEPORTIVA EXTRAESCOLAR ...

Por otra parte, encontramos la necesidad de competencia a través de la cual el alumno se siente capaz de poder realizar adecuadamente todo aquello que se proponga, es decir, tener la sensación de poder resolver aquellas tareas que le son planteadas con altas probabilidades de éxito y con gran eficacia (Timo, Sami, Anthony y Jarmo, 2016).

A la hora de percibir su nivel competencia en las clases de EF, los alumnos se comparan con el resto de los compañeros, y en función de los resultados que obtienen los demás, califican su nivel (orientación al ego), sin embargo, los alumnos deberían medir su nivel de competencia en función de sus logros personales, comparando sus resultados con los que ellos mismos han obtenido previamente, estableciendo así su mejora personal (orientación a la tarea). De este modo cuando el alumno se encuentra orientado al ego, centrándose únicamente en superar a los demás, comparándose constantemente con estos, su percepción de competencia comienza a peligrar, debido a que esta pierde su validez y fiabilidad (Nicholls, 1989). Este aspecto tiene consecuencias negativas para el alumno, ya que comienza a desmotivarse y por lo tanto, a sentirse incompetente, no consiguiendo controlar los resultados, además de infravalorar la propia actividad (Deci y Ryan, 2000). De ahí la importancia del clima motivacional percibido que los profesores generan en las clases de EF ya que, de esta manera, si las señales situacionales son fuertes, se puede llegar a anular o modificar la orientación disposicional del alumno a lo hora de realizar las tareas (Granero-Gallegos y Baena-Extremera, 2014), favoreciendo así su capacidad de percibir si es competente en las clases.

Por último, la tercera necesidad psicológica básica es la de relación con los demás, la cual se basa en que los estudiantes necesitan sentirse conectados con sus profesores, compañeros y con la propia escuela (Deci y Ryan, 1985, 2000). Hay que recordar que el contexto social es muy importante para los alumnos, ya que la actitud tanto de profesores como de compañeros va a influir en que el alumno satisfaga las otras dos NPB tratadas anteriormente (Xiang, Agbuga, Liu y McBride, 2017). El factor social que más se ha estudiado en esta necesidad psicológica, es la relación del alumno con su profesor, argumentando que tanto la cooperación (Ntoumanis, 2001) como el apoyo a la autonomía y la generación de oportunidades para establecer conexiones sociales en las clases de EF son los aspectos en los que más enfatiza el profesor de EF durante sus clases (Standage et al., 2005).

Esta necesidad psicológica es muy importante para el desarrollo del alumno en la adolescencia, quedando demostrado en distintos estudios que el sentirse conectado socialmente es un predictor mucho más fuerte de la motivación autodeterminada que las otras dos NPB (Cox y Williams, 2008; Standage, Duda y Ntoumanis, 2003).

Cuando los alumnos satisfacen estas tres NPB incrementan su compromiso deportivo (García, Sánchez, Leo, Sánchez y Amado, 2011), rendimiento y aprendizaje, siendo el centro educativo el contexto ideal que debe conseguir satisfacerlas (Deci y Ryan, 1985). Además, su satisfacción también favorece otros aspectos como la autoestima, la satisfacción y la diversión (Balaguer, Castillo y Duda, 2008; Calvo, Miguel, Marcos, Oliva y Alonso, 2012), así como su bienestar general (Sheldon y Elliot, 1999).

En cuanto a la relación entre las NPB y la variable género, la literatura demuestra que los chicos perciben mayores niveles de autonomía y competencia durante las clases de EF, mientras que las chicas, establecen más y mejores conexiones sociales con sus compañeros (Gómez, Hernández, Martínez y Gámez, 2014). Sin embargo, también existen también otros estudios donde no se han encontrado diferencias en función de esta variable (AguadoGómez et al., 2016). 
Por otro lado, la satisfacción de NPB en las clases de EF, gracias al clima generado y a las actividades propuestas por el profesor, va a influir sobre el miedo al fallo y el rechazo a la realización de las tareas en las clases de EF (Conroy, Poczwardowski y Henschen, 2001), ya que las tareas que componen las clases, se dan en un contexto de logro en el que los alumnos quieren ganar y superar a sus compañeros, pudiendo demostrar ante los demás sus habilidades y destrezas, pero en ocasiones puede tener consecuencias negativas para el alumnado, generando inseguridad, ansiedad, estrés y el rechazo a la práctica deportiva entre los alumnos menos destacados en el ámbito motriz, debido al miedo a fallar y pasar vergüenza por la reacción de los compañeros (Silveira y Moreno, 2015).

Es por ello, que algunos alumnos con un nivel bajo de destreza motriz pueden llegar a sentir cierto temor o nerviosismo durante las clases, debido al estrés que les genera esta situación de observación (Silveira y Moreno-Murcia, 2015). Como consecuencia de este sentimiento, el alumno buscará excusas para no realizar la tarea o reducirá su grado de implicación en el desarrollo de la misma (Pensgaard y Roberts, 2000; Ruiz-Sánchez, Gómez-López, Granero-Gallegos y González, 2017). La competitividad que existe a estas edades y la evaluación recibida por sus acciones produce que los alumnos tengan miedo a fallar o a tomar decisiones durante el desarrollo de las clases.

El miedo al fallo es definido como una tendencia estable para anticipar la vergüenza y la humillación después del fallo (Conroy, Willow y Metzler, 2002). Este miedo a fallar aparece cuando los alumnos dejan que su conducta se vea influenciada por los demás, tratando constantemente de satisfacer al resto y temiendo sus reacciones por posibles fallos en la realización de las actividades (Moreno-Murcia, Conte, Silveira y Ruiz, 2014). Por lo que según Conroy et al. (2002) el miedo al fallo provoca la valoración de las consecuencias negativas (ansiedad o depresión) que tienen para el bienestar las posibles emociones que puedan llegar a sentir los alumnos después de no realizar correctamente una tarea delante de sus compañeros o profesores (Conroy et al., 2001; Sagar, Lavallee y Spray, 2007).

Este miedo genera una actitud negativa hacia las clases de EF, en las que los alumnos no se esfuerzan en realizar las tareas adecuadamente, poniendo constantemente excusas para no realizar los ejercicios, convirtiéndose en una estrategia de autodefensa (Chen, Chen, Lin, Kee y Shui, 2009). Según Berglas y Jones (1978) esta estrategia de autodefensa la llevan a cabo los alumnos para proteger su autoestima, ya que al no realizar los ejercicios evitan posibles situaciones en las que puedan llegar a sentir vergüenza delante de sus compañeros por la realización incorrecta de una tarea, o bien por la posible valoración negativa realizada por el profesor delante del resto de la clase.

Este miedo al fallo les será útil únicamente a corto plazo, debido a que durante estas clases en las que se niegan a realizar las tareas no tendrán que estar pendientes de los posibles comentarios del resto de compañeros, sin embargo, esta actitud tendrá influencias negativas tanto para su motivación como para su bienestar, a largo plazo (Zuckerman y Tsai, 2005).

Con respecto a la relación del miedo al fallo y la variable género, existen estudios en los que no se encuentran diferencias significativas (Silveira y Moreno, 2015). Sin embargo, encontramos otras investigaciones en las que los resultados muestran que las alumnas son más propensas que los chicos a inventar excusas para no realizar las actividades propuestas en clase de EF (Chen et al., 2009), prefiriendo, independientemente del sexo, poner excusas y reducir el nivel del esfuerzo en la realización de las tareas propuestas 

RELACIÓN CON EL MIEDO AL FALLO SEGÚN EL GÉNERO Y LA PRÁCTICA FÍSICO-DEPORTIVA EXTRAESCOLAR ...

por el profesor. Analizando cada una de las causas aversivas del miedo al fallo, se ha demostrado que para los chicos el principal miedo es perder su valor social, es decir, que el fracaso afecte negativamente a su relación con otros importantes, mientras que las chicas creen por otro lado que el fracaso es indicador de tener poca habilidad y control en el desempeño de las actividades, afectando a su autoestima (Sagar, Boardley y Kavussanu, 2010). En el ámbito del deporte, se ha demostrado que las causas aversivas de miedo al fallo están más acentuadas en los chicos que en las chicas, siendo la vergüenza y la devaluación de uno mismo las más expresadas por las mujeres, y el futuro incierto, perder el interés de los demás y la perturbación a otros importantes las de los hombres (RuizSánchez et al., 2017; Munuera, Gómez-López, Granero-Gallegos, y Sánchez-Alcaraz, 2018). Aunque son escasos los resultados encontrados sobre el miedo al fallo según el género de los deportistas, los estudios en general revelan que los chicos presentan un mayor miedo a equivocarse que las chicas (Moreno-Murcia, Martínez-Galindo, Belando y Conte, 2010; Moreno-Murcia, Ferriz, Belando y Conte, 2011).

En cuanto a los estudios que han abordado la relación entre las NPB y el miedo al fallo de los estudiantes en las clases de EF, debemos afirmar que son inexistentes en nuestro país y escasos fuera del mismo. Los resultados de estas investigaciones reflejan que este rechazo a la realización de las actividades durante las sesiones por el miedo a lo que puedan opinar los demás si fallan se asocia con una percepción de incompetencia durante las clases (Smith, Sinclair y Chapman, 2002; Zuckerman y Tsai, 2005). Es más, se ha comprobado como este miedo al fallo se ve reducido en aquellos alumnos que perciben mayor autonomía por parte del profesor durante sus clases, ya que esta oportunidad de poder realizar las tareas a su manera les da seguridad y afecta positivamente a la autoestima del alumno que no se ve tan mermada si no consigue el éxito (Conroy y Coatsworth, 2007).

Por otra parte, aquellos a los que el clima motivacional planteado por el profesor les favorece el poder relacionarse y establecer conexiones afectivas con el resto de los compañeros, tienen niveles más bajos de miedo al fallo, ya que se sienten más seguros a la hora de realizar las tareas debido a la confianza que tienen con los demás, evitando así la posibilidad de pensar que les van a criticar y por tanto de sentir vergüienza sino realizan correctamente las tareas (Seifriz, Duda y Chi, 1992).

Finalmente, señalar la relevancia de los resultados de este trabajo, puesto que la mayoría de la literatura revisada refleja que ambas variables se han analizado por separado (NPB y miedo al fallo), y las escasas investigaciones que se han realizado relacionando ambas variables han reflejado la importancia de la figura docente en la satisfacción de las NPB de los estudiantes y su relación con el miedo a equivocarse en las clases de EF, pero no han aportado información relacionada con el hábito de práctica físico-deportiva extraescolar de los estudiantes. Además, los resultados que se obtengan ayudarán a los profesores de EF a conocer en qué aspectos deben incidir y reforzar según el género del estudiante y su hábito de práctica deportiva extraescolar, y que tipo de clima motivacional deben favorecer con el fin de potenciar la satisfacción de las NPB y disminuir el miedo a equivocarse en las clases de EF. De esta forma, fomentarán el interés y la atracción de los estudiantes por las clases de EF y su adherencia a la práctica de actividades físico-deportivas.

Por lo tanto, los objetivos del estudio fueron: describir el nivel de satisfacción de las NPB y la percepción de miedo al fallo de los estudiantes de secundaria en las clases de 
Estudios Pedagógicos XLVII N 1: 303-322, 2021

SATISFACCIÓN DE LAS NECESIDADES PSICOLÓGICAS BÁSICAS EN LAS CLASES DE EDUCACIÓN FÍSICA Y SU RELACIÓN CON EL MIEDO AL FALLO SEGÚN EL GÉNERO Y LA PRÁCTICA FÍSICO-DEPORTIVA EXTRAESCOLAR ...

EF y analizar su relación y las diferencias que existen en función de las variables género y práctica deportiva del estudiante fuera del contexto educativo.

\section{MATERIAL Y MÉTODO}

\subsection{PARTICIPANTES}

La muestra estuvo compuesta por un total de 464 estudiantes de los cuales 238 eran hombres $(51,3 \%)$ y 226 mujeres $(48,7 \%)$ de edades comprendidas entre 12 y 17 años $(M=14,08$; DT= 1,53), pertenecientes a diversos centros públicos de ESO de la Región de Murcia. Del total de la muestra, un $61 \%$ afirmaron que practicaban actividades físico-deportivas en horario extraescolar y un $39 \%$ que no lo hacían.

\subsection{INSTRUMENTOS}

Se utilizó la versión española adaptada a la EF (Moreno-Murcia, González-Cutre, Chillón y Parra, 2008; Menéndez Santurio y Fernández-Río, 2018) del test Necesidades Psicológicas Básicas (Basic Psychological Needs in Exercise Scale, BPNES; Vlachopoulos y Michailidou, 2006; Vlachopoulos, Katartzi y Kontou, 2011). La escala está compuesta por 12 ítems (4 por factor) que evalúan la autonomía (e.g. "Pienso que la forma en que se imparte la educación física es tal y como a mí me gusta”), competencia (e.g. "Creo que mejoro incluso en las tareas que la mayoría de los compañeros considera difíciles") y relación (e.g. "Las relaciones con mis compañeros de clase son muy amistosas"). Cada ítem se iniciaba con la frase "En mis clases de educación física...". Las respuestas se recogieron en una escala tipo Likert de 5 puntos que oscilaba desde totalmente en desacuerdo (1) a totalmente de acuerdo (5). En este estudio, el análisis de consistencia interna ha resultado satisfactorio en las diferentes subescalas (satisfacción de autonomía, a = ,71; satisfacción de competencia, a = ,71; satisfacción de relación con los demás, $\mathrm{a}=, 82$ ).

Además, se utilizó la versión larga del inventario validada al contexto educativo español (Moreno-Murcia, Silveria y Conte, 2013) Inventario de Evaluación del Error en el Rendimiento (Performance Failure Appraisal Inventory, PFAI; Conroy et al., 2002), adaptada a partir de la primera validación realizada en el ámbito deportivo (Moreno-Murcia y Conte, 2011). La escala consta de 25 ítems, agrupados en cinco dimensiones: miedo de experimentar vergüenza (ej.: "Cuando me equivoco, me da vergüenza si los demás están allí para verlo"), miedo a la devaluación de uno mismo (ej.: "Cuando no tengo éxito, me siento menos valioso que cuando tengo éxito"), miedo de tener un futuro incierto (ej.: "Cuando me equivoco, creo que mis planes para el futuro cambiarán"), miedo de perder el importante interés de los demás (ej.: "Cuando no tengo éxito, algunas personas no se muestran interesadas por mî"), y el temor de perturbar a otros importantes (ej.: "Cuando me equivoco, esto disgusta a la gente que me importa"). Cada ítem se iniciaba con la frase "En la práctica de la clase de EF...". Las respuestas se recogieron en una escala tipo Likert de 5 puntos que oscilaba desde (1) no lo creo nada a (5) lo creo al $100 \%$. En este estudio, el análisis de consistencia interna ha resultado satisfactorio en las diferentes subescalas (miedo de experimentar vergüenza, $a=, 83$; miedo a la devaluación de uno mismo, $\mathrm{a}=, 69$; miedo de tener un futuro incierto, $\mathrm{a}=, 70$; miedo de perder $\mathrm{C}$ el importante interés de los demás, $\mathrm{a}=, 81$; temor de perturbar a otros importantes, $\mathrm{a}=, 76$ ). 


\subsection{PROCEDIMIENTO}

Para poder asistir a los centros educativos y realizar el trabajo de campo, se obtuvo autorización de la Dirección de cada Centro, Consejo Escolar y profesores de EF de los cursos asignados en la toma de datos, y de los padres de los propios estudiantes. Previamente a la aplicación del instrumento, se informó al alumnado de la finalidad del estudio, de su voluntariedad y anonimato. Para la cumplimentación del cuestionario se requirió una media de 20 minutos de la clase de $\mathrm{EF}$, variando ligeramente en función de la edad. Todo el trabajo de campo se llevó a cabo siempre en presencia de dos encuestadores y del profesor de EF.

\subsection{ANÁLISIS DE DATOS}

En primer lugar, fueron calculados los estadísticos descriptivos, correlación entre las subescalas, consistencia interna de cada subescala (alfa de Cronbach) y los índices de asimetría y curtosis, siendo estos entre -1.26 y 1.31 , lo que indica semejanza con la curva normal de forma univariada. Para calcular las diferencias en función de la variable sexo y de la práctica deportiva se realizó un análisis de diferencia de medias mediante la prueba de la $\mathrm{T}$ de Student para muestras independientes en la que se tuvo en cuenta la prueba de Levene, de homogeneidad de las varianzas. Se calculó, asimismo, el tamaño del efecto (eta parcial al cuadrado) para cuantificar la relación -diferencias- entre las variables en que se hallaron diferencias estadísticamente significativas. El cálculo estadístico se realizó con el paquete SPSS en su v.22 para Windows.

\section{RESULTADOS}

\subsection{ANÁLISIS DESCRIPTIVO Y DE CORRELACIÓN}

Los valores descriptivos de distintas variables estudiadas se muestran en la Tabla 1. En relación a la BPNES, los resultados hallados manifiestan un valor medio más alto en la relación con los demás $(M=4.10)$, y la autonomía la de valor medio más bajo $(M=3.13)$. En lo que respecta al PFAI, el miedo a experimentar vergüenza es la que puntuó más alto $(M=2.41)$, seguida del miedo a la devaluación de uno mismo $(M=2.06)$, siendo, el miedo a tener un futuro incierto la dimensión que presentó los valores medios más bajos $(M=1.64)$.

$\mathrm{El}$ análisis de correlación pone de manifiesto la positiva y significativa, desde el punto de vista estadístico, relación entre los tres factores de la BPNES, así como entre los factores del PFAI, destacando la correlación entre el miedo a experimentar vergüenza y el miedo a la devaluación de uno mismo. En lo que respecta a la correlación entre las dos escalas, se encontró ausencia de correlación estadísticamente significativa entre la autonomía y los factores del miedo, a excepción del miedo a perder el interés de los demás, cuya relación resultó negativa. También las correlaciones entre la competencia y los factores del miedo al fallo son negativas, resaltado las relaciones con el miedo a tener un futuro incierto y a perder el importante interés de otros; no obstante, la correlación presentó un coeficiente bajo. Finalmente, hay que destacar que el factor denominado relación con los demás de la BPNES mostró correlaciones estadísticamente significativas y negativas con las cinco 
Estudios Pedagógicos XLVII N $1:$ 303-322, 2021

SATISFACCIÓN DE LAS NECESIDADES PSICOLÓGICAS BÁSICAS EN LAS CLASES DE EDUCACIÓN FÍSICA Y SU RELACIÓN CON EL MIEDO AL FALLO SEGÚN EL GÉNERO Y LA PRÁCTICA FÍSICO-DEPORTIVA EXTRAESCOLAR ...

dimensiones del PFAI, resaltado el miedo a perder el importante interés de otros. Estos resultados ponen de manifiesto la importancia de las relaciones sociales en la EF en estas edades de Secundaria.

Tabla 1. Descriptivos, consistencia interna y correlaciones de las subescalas del BPNES y del PFAI

\begin{tabular}{|c|c|c|c|c|c|c|c|c|c|c|c|}
\hline Subescalas & $M$ & $D T$ & $\alpha$ & 1 & 2 & 3 & 4 & 5 & 6 & 7 & 8 \\
\hline \multicolumn{12}{|l|}{ BPNES } \\
\hline 1. Autonomía & 3.13 & .82 & .71 & & $.51 * *$ & $.38 * *$ & -.07 & -.08 & -.01 & $-.12 * *$ & -.05 \\
\hline 2. Competencia & 3.62 & .83 & .71 & & & $.50 * *$ & -.08 & $-.12 *$ & $-.16 * *$ & $-.17 * *$ & $-.12 *$ \\
\hline 3. Relación con los demás & 4.10 & .89 & .82 & & & & $-.17 * *$ & $-.19 * *$ & $-.23 * *$ & $-.29 * *$ & $-.19 * *$ \\
\hline \multicolumn{12}{|l|}{ PFAI (Miedo a...) } \\
\hline 4. Experimentar vergüenza & 2.41 & .86 & .80 & & & & & $.60 * *$ & $.41 * *$ & $.55^{* *}$ & $.53 * *$ \\
\hline 5. Devaluación de uno mismo & 2.06 & .74 & .58 & & & & & & $.53 * *$ & $.46^{* *}$ & $.49 * *$ \\
\hline 6. Tener un futuro incierto & 1.64 & .75 & .69 & & & & & & & $.36^{* *}$ & $.52 * *$ \\
\hline 7. Perder el importante interés de otros & 1.70 & .74 & .78 & & & & & & & & $.46^{* *}$ \\
\hline 8. Perturbar a otros importantes & 1.87 & .79 & .70 & & & & & & & & \\
\hline
\end{tabular}

Nota. $* \rho<.05 ; * * \rho<.01 . M=$ Media; $D T=$ Desviación típica; $\alpha=$ alfa de Cronbach.

\subsection{DIFERENCIAS SEGÚN GÉNERO}

Con el objeto de analizar las diferencias en función de la variable género, se realizó la prueba $\mathrm{T}$ de Student. Los datos expuestos en la Tabla 2 indicaron solamente diferencias en la dimensión de competencia, en la que los chicos mostraron una puntuación media más alta que las chicas ( $M=3.75$ y $M=3.48$ respectivamente). No se obtuvieron diferencias estadísticamente significativas en ninguna de las dimensiones del PFAI. 
SATISFACCIÓN DE LAS NECESIDADES PSICOLÓGICAS BÁSICAS EN LAS CLASES DE EDUCACIÓN FISICA Y SU RELACIÓN CON EL MIEDO AL FALLO SEGÚN EL GÉNERO Y LA PRÁCTICA FÍSICO-DEPORTIVA EXTRAESCOLAR ...

Tabla 2. Prueba T de Student para muestras independientes según el género

\begin{tabular}{|l|c|c|c|c|c|c|}
\hline \multicolumn{1}{|c|}{ Subescalas } & $\begin{array}{c}\text { Chicos } \\
\boldsymbol{M}(\boldsymbol{D T})\end{array}$ & $\begin{array}{c}\text { Chicas } \\
\boldsymbol{M}(\boldsymbol{D} \boldsymbol{T})\end{array}$ & $\mathbf{t}$ & gl & $\boldsymbol{p}$ & $\begin{array}{c}\text { Eta parcial } \\
\text { al cuadrado }\end{array}$ \\
\hline BPNES & & & & & & \\
\hline 1. Autonomía & $3.18(.84)$ & $3.08(.79)$ & 1.33 & 462 & .184 & .01 \\
\hline 2. Competencia & $3.75(.82)$ & $3.48(.82)$ & 3.47 & 462 & .001 & .03 \\
\hline 3. Relación con los demás & $4.13(.89)$ & $4.07(.89)$ & .72 & .462 & .469 & .00 \\
\hline PFAI (Miedo a...) & & & & & & .00 \\
\hline 4. Experimentar vergüenza & $2.37(.84)$ & $2.44(.89)$ & -.90 & 462 & .368 & .00 \\
\hline 5. Devaluación de uno mismo & $2.08(.75)$ & $2.03(.72)$ & .71 & 462 & .478 & .00 \\
\hline 6. Tener un futuro incierto & $1.67(.79)$ & $1.59(.71)$ & 1.13 & 462 & .258 & .00 \\
\hline $\begin{array}{l}\text { 7. Perder el importante interés } \\
\text { de otros }\end{array}$ & $1.75(.77)$ & $1.64(.70)$ & 1.66 & 462 & .097 & .01 \\
\hline 8. Perturbar a otros importantes & $1.89(.76)$ & $1.83(.81)$ & .77 & 462 & .440 & .00 \\
\hline
\end{tabular}

Nota. $M=$ media; $D T=$ desviación típica; $* * *=p<, 000$.

\subsection{DIFERENCIAS SEGÚN LA PRÁCTICA DEPORTIVA EXTRAESCOLAR}

Para comprobar las diferencias entre el alumnado que practica actividad física y deportiva fuera del centro y los que no, se llevó a cabo la prueba T de Student. En este caso, se hallaron diferencias estadísticamente significativas en los tres factores de la BPNES, y tanto en la autonomía, competencia, como relación con los demás, la puntuación media fue más alta entre quienes sí practican actividad deportiva en su tiempo libre fuera del centro que quienes no son practicantes ( $M=3.21, M=3.78$ y $M=4.20$ respectivamente). El tamaño del efecto fue más alto en la subescala competencia. Respecto al PFAI, solo en el caso de miedo a tener un futuro incierto se encontraron diferencias estadísticamente significativas, y en este caso quienes no práctica actividad deportiva fuera del centro alcanzaron valores medios más altos que quienes sí practican ( $M=1.75$ y $M=1.56$ respectivamente). 
Estudios Pedagógicos XLVII N $1:$ 303-322, 2021

SATISFACCIÓN DE LAS NECESIDADES PSICOLÓGICAS BÁSICAS EN LAS CLASES DE EDUCACIÓN FÍSICA Y SU RELACIÓN CON EL MIEDO AL FALLO SEGÚN EL GÉNERO Y LA PRÁCTICA FÍSICO-DEPORTIVA EXTRAESCOLAR ...

Tabla 3. Diferencias, según grupos si practica o no deporte regularmente fuera del horario escolar, en las diferentes subescalas estudiadas

\begin{tabular}{|c|c|c|c|c|c|c|}
\hline \multirow[b]{2}{*}{ Subescalas } & \multicolumn{2}{|c|}{ Practica deporte } & \multirow[b]{2}{*}{$\mathbf{t}$} & \multirow[b]{2}{*}{ gl } & \multirow[b]{2}{*}{$p$} & \multirow{2}{*}{$\begin{array}{l}\text { Eta parcial } \\
\text { al cuadrado }\end{array}$} \\
\hline & $\begin{array}{l}\text { Sí (n=283) } \\
M \text { (DT) }\end{array}$ & $\begin{array}{c}\text { No }(\mathrm{n}=181) \\
M(D T)\end{array}$ & & & & \\
\hline \multicolumn{7}{|l|}{ BPNES } \\
\hline 1. Autonomía & $3.21(.81)$ & $2.99(.81)$ & 2.89 & 462 & .004 & .02 \\
\hline 2. Competencia & $3.78(.76)$ & $3.37(.86)$ & 5.15 & 348,773 & .000 & .06 \\
\hline 3. Relación con los demás & $4.20(.84)$ & $3.94(.94)$ & 3.08 & 462 & .003 & .02 \\
\hline \multicolumn{7}{|l|}{ PFAI (Miedo a...) } \\
\hline 4. Experimentar vergüenza & $2.40(.85)$ & $2.41(.87)$ & -.04 & 462 & .972 & .00 \\
\hline 5. Devaluación de uno mismo & $2.00(.71)$ & $2.14(.77)$ & 1.89 & 462 & .060 & .01 \\
\hline 6. Tener un futuro incierto & $1.56(.69)$ & $1.75(.83)$ & 2.62 & 335,318 & .009 & .02 \\
\hline $\begin{array}{l}\text { 7. Perder el importante interés } \\
\text { de otros }\end{array}$ & $1.65(.73)$ & $1.77(.75)$ & 1.58 & 462 & .115 & .01 \\
\hline 8. Perturbar a otros importantes & $1.82(.77)$ & $1.94(.81)$ & 1.57 & 462 & .118 & .01 \\
\hline
\end{tabular}

\section{DISCUSIÓN Y CONCLUSIONES}

Los objetivos del estudio fueron describir el nivel de satisfacción de las NPB y la percepción de miedo al fallo de los estudiantes de secundaria en las clases de EF y analizar su relación y las diferencias que existen en función de las variables género y práctica deportiva del estudiante fuera del contexto educativo.

El análisis descriptivo muestra que la NPB que afirman tener más satisfecha es la de relacionarse con los demás, mientras que la autonomía es la que menos se satisface. Estos resultados reflejan la importancia de las relaciones sociales que se potencian en las clases de EF y coinciden con otros estudios anteriores realizados también en el contexto educativo (Gómez et al., 2014; Leptokaridou, Vlachopoulos y Papaioannou, 2015; Moreno, Cervelló, Montero, Vera y García, 2012; Moreno, Jiménez, Gil, Aspano, y Torrero, 2011; Moreno et al., 2008; Méndez-Giménez, Fernández-Río y Cecchini Estrada, 2013).

Por lo tanto, y a partir de los resultados, sería conveniente que el profesorado continúe fomentando las tareas de enseñanza-aprendizaje en las que se potencien las relaciones sociales de los alumnos tanto con el profesorado como con sus compañeros. Además, tal y como se refleja en la literatura, la satisfacción de dicha NPB es fundamental para la satisfacción de las otras necesidades de autonomía y competencia (Xiang et al., 2017), así como para el buen desarrollo del alumno, ya que, según los estudios, la necesidad de relación con los demás es un predictor muy potente de la motivación autodeterminada (Cox y Williams, 2008; Standage et al., 2003). Además, los resultados hallados reflejaron que sería conveniente que los profesores fomentaran en mayor medida la autonomía 
SATISFACCIÓN DE LAS NECESIDADES PSICOLÓGICAS BÁSICAS EN LAS CLASES DE EDUCACIÓN FÍSICA Y SU RELACIÓN CON EL MIEDO AL FALLO SEGÚN EL GÉNERO Y LA PRÁCTICA FÍSICO-DEPORTIVA EXTRAESCOLAR ...

y la competencia durante las clases de EF; es más, según la literatura especializada la satisfacción de ambas necesidades tienen un papel importante en el grado de satisfacción y de diversión de los estudiantes con las clases de EF (Baena-Extremera, Gómez-López, Granero-Gallegos y Martínez-Molina, 2016; Gómez-López et al., 2015), puesto que influirá positivamente en su motivación intrínseca (Cox y Williams, 2008; Goudas et al., 1994; Moreno-Murcia et al., 2013; Standage et al., 2005).

La literatura refleja que esta conducta autodeterminada del alumno (Deci y Ryan, 1985, 2000), fomentará una mayor adherencia al deporte (Harris et al., 2009) y un estilo de vida activo (Chatzisarantis y Hagger, 2009; Lim y Wang, 2009; Zhang et al., 2012), siendo ambos aspectos necesarios en la vida del estudiante para su bienestar físico, mental y social, sobre todo en la adolescencia, ya que es una etapa en la que las personas establecen sus hábitos de vida, siendo muy difícil su variación una vez finalizada dicha etapa (Heaven, 1996).

Por otra parte, en contraste con los resultados obtenidos, no coincidimos con los hallados anteriormente por otros estudios realizados en muestras con edades superiores a la analizada. Gunnell, Crocker, Wilson, Mack y Zumbo (2013) con estudiantes universitarios, hallaron que la NPB que más sentían tener satisfecha fue la autonomía, y la que menos la relación con los demás. Goulimaris, Mavridis, Genti y Rokka (2014), con practicantes de danza, con edades comprendidas entre los 40 y 70 años, obtuvieron que la NPB que afirmaron tener más satisfecha fue relacionarse con los demás, mientras que la que menos la competencia.

En cuanto al análisis descriptivo de la percepción de miedo al fallo, se demostró que el miedo a experimentar vergüienza y a la devaluación de uno mismo fueron las causas aversivas que más mencionaron los estudiantes, mientras que el miedo a tener un futuro incierto fue la que menos, coincidiendo de este modo con otros estudios anteriores, de características muy similares, pertenecientes al ámbito educativo, cuyas edades oscilaron entre los 12 y 17 años (De Castella, Byrne y Covington, 2013; Munuera et al., 2018; Silveira y Moreno, 2015).

Por lo tanto, a tenor de los resultados del estudio, es recomendable que el profesor de EF incida en la disminución de las distintas dimensiones del miedo a equivocarse del estudiante ya que, como se ha demostrado en la literatura, este miedo genera una actitud negativa hacia la práctica de actividad física (Chen et al., 2009) que puede llegar a provocar, incluso, el abandono deportivo (Gil, Campos, Jordán y Díaz, 2012) y generar a medio y largo plazo consecuencias negativas para el bienestar del alumno y su motivación (Conroy et al., 2002; Zuckerman y Tsai, 2005). Para ello, y a partir de lo hallado en otros estudios, es clave el feedback empleado por el profesor durante las clases de EF, ya que este puede definir un clima motivacional adecuado durante las sesiones, además de las consecuencias positivas que puede llegar a tener en la motivación y la percepción de competencia de los alumnos en las clases de EF (Amorose y Smith, 2003; Nicaise, Cogerino, Bois y Amorose, 2006).

Son muchos los estudios que han demostrado que la causa aversiva que más se pronuncia es el miedo a experimentar vergüenza, por ello es considerado como la base del resto de dimensiones de miedo al fallo (Conroy, 2004; Ruiz-Sánchez et al., 2017; Sagar et al., 2010; Sagar, Busch y Jowett, 2010; Sagar y Stoeber, 2009), coincidiendo así con la principal causa aversiva obtenida en el presente estudio, a pesar de que en estos casos estos estudios revisados se realizaron en el ámbito deportivo y con participantes con edades superiores a 16 años. 
El análisis diferencial de la satisfacción de las NPB y el género de los estudiantes reflejó diferencias estadísticamente significativas en la dimensión de competencia, mostrándose un mayor sentimiento de satisfacción de esta necesidad en los chicos. Estos resultados coinciden con otros estudios anteriores como los llevados a cabo por Cervelló, Jiménez, del Villar, Ramos y Santos-Rosa (2004); Leversen, Danielsen, Birkeland y Samdal (2012), en el ámbito educativo con alumnos de edades similares. Por otra parte, Brunet y Sabiston (2009), hallaron que los chicos tenían valores superiores de satisfacción de las tres NPB, lo que puede deberse a que la edad de los estudiantes de la muestra osciló entre los 17 y los 23 años, además de que el instrumento de medida utilizado fue diferente. Además, también existe otro estudio realizado con una muestra similar, dentro del ámbito educativo y perteneciente a la etapa secundaria, cuyo resultado también reflejó que además de sentirse más competentes, los estudiantes percibieron mayor autonomía en el desarrollo de las actividades de clase (Gómez et al., 2014). Por otro lado, también existen otros trabajos realizados también en el contexto educativo de secundaria (AguadoGómez et al., 2016; Gutiérrez, García-López, Hastie, y Calderón, 2013; Orkibi y Ronen, 2017) donde no se encontraron diferencias significativas en función de esta variable sociodemográfica.

En cuanto a la práctica físico-deportiva extraescolar, se comprobó que los alumnos que practicaban deporte fuera del centro educativo tuvieron valores significativamente superiores en la satisfacción de las tres NPB, coincidiendo de este modo con el estudio de Leversen et al. (2012), con estudiantes de 15 y 16 años. Por lo tanto, y a tenor de los resultados, es fundamental fomentar un estilo de vida saludable y activo desde el entorno educativo con la figura del profesor de EF y desde el ámbito familiar.

El estudio realizado por Taylor, Ntoumanis, Standage y Spray (2010), con una muestra de estudiantes con edades comprendidas entre los 11 y 16 años, reflejó que aquellos que se sentían más competentes a la hora de realizar las tareas propuestas en clase eran más activos y como consecuencia, tuvieron índices superiores de práctica extraescolar.

Con respecto a la percepción del miedo al fallo en función del género del estudiante, no se han encontrado diferencias estadísticamente significativas. De este modo los resultados coincidieron con otros estudios realizados en secundaria, donde tampoco se hallaron diferencias estadísticamente significativas en función de esta variable sociodemográfica (Munuera et al., 2018; Silveira y Moreno, 2015). Asimismo, en el contexto deportivo también encontramos estudios como el de Correia, Rosado, Serpa y Ferreira (2017), con resultados similares en participantes con edades parecidas a las del estudio.

Por otro lado, otro estudio realizado con deportistas en edad escolar demostró que los chicos tenían valores del miedo al fallo significativamente superiores en todas las dimensiones excepto en el miedo a tener un futuro incierto, donde fueron las chicas las que tuvieron valores superiores (Amjad, Irshad y Gul, 2018). Siguiendo con estudios realizados en el ámbito deportivo, más concretamente con jugadores de balonmano, se demostró que, en las chicas, el miedo a experimentar vergüenza y a la devaluación de uno mismo, son las causas aversivas que más expresan, mientras que, el miedo a un futuro incierto, a perder el interés de los demás y a la perturbación de otros importantes, son las más proclives entre los chicos (Ruiz-Sánchez et al., 2017).

Resaltar que la mayoría de los estudios revisados coinciden de forma general en que los chicos tienen niveles superiores de miedo al fallo en el ámbito deportivo que las chicas (McGregor y Elliot, 2005; Moreno-Murcia et al., 2010, 2011), mientras que, en el 

RELACIÓN CON EL MIEDO AL FALLO SEGÚN EL GÉNERO Y LA PRÁCTICA FÍSICO-DEPORTIVA EXTRAESCOLAR ...

ámbito educativo, son las chicas las más propensas a inventar excusas para no realizar las actividades propuestas en clase de EF (Chen et al., 2009).

En cuanto a la variable de práctica físico-deportiva extraescolar, solamente se pudo demostrar que los estudiantes que afirmaron no practicar actividad físico-deportiva fuera del contexto escolar tuvieron más miedo a tener un futuro incierto que los que si practicaban. Por lo tanto, y a tenor de los resultados, resulta importante que el profesor utilice las estrategias necesarias para disminuir lo máximo posible las causas aversivas del miedo a equivocarse, ya que esto podría favorecer la participación de los alumnos durante las clases de EF, teniendo así, según la literatura, más posibilidades de fomentar el placer y la satisfacción por la práctica deportiva (Baena-Extremera, Gómez-López, Granero-Gallegos y Ortiz-Camacho, 2015). Dentro de este aspecto, el estudio realizado por Vilhjalmsson y Thorlindsson (1998) reflejó que resulta crucial atender a las diferencias de oportunidades que existen entre niños y niñas para poder realizar actividad físico-deportiva extraescolar, debido principalmente a la falta de acceso, y demanda de elección entre las chicas y a la mentalidad competitiva que existe aún en muchas escuelas deportivas que a veces no son compatibles con las habilidades físicas de las niñas.

Finalmente, los resultados de la correlación entre las dos variables analizadas demostraron de manera general una asociación negativa entre las NPB y la percepción de miedo al fallo. La NPB autonomía no se relacionó de manera significativa con ninguna de las dimensiones del miedo al fallo, a excepción de la de perder el interés de otros. La literatura ha demostrado que es fundamental que el profesor otorgue cada vez mayores niveles de autonomía a los alumnos durante las clases de EF, ya que provocará que los estudiantes aumenten su motivación intrínseca (Goudas et al., 1994), consiguiendo así mayor adherencia a la actividad física regular (Harris et al., 2009; Sánchez-Alcaraz, Murcia Gálvez, Alfonso-Asencio y Hellín-Martínez, 2020), y adquiriendo por tanto, un estilo de vida activo (Chatzisarantis y Hagger, 2009; Lim y Wang, 2009; Zhang et al., 2012). Sin embargo, atendiendo a otros estudios realizados con estudiantes de 15 años, se comprobó como la autonomía si mantiene una relación positiva y significativa con el miedo a tener un futuro incierto, el miedo a perturbar a otros importantes, y el miedo a perder el interés de otros. Además, en este mismo estudio, también se mostró una relación significativa e inversa con el miedo a experimentar vergüenza (Moreno-Murcia et al., 2013). De manera general, Conroy y Coastworth (2007) demostraron que el miedo al fallo se vio reducido en aquellos estudiantes que percibieron mayor autonomía por parte del profesor durante las clases, ya que, al tener la oportunidad de hacer las tareas de clase con libertad, incrementaron su seguridad y autoestima.

Por otro lado, la sensación de competencia en las clases de EF se relacionó negativa y significativamente con el miedo a tener un futuro incierto y perder el interés de otros, mientras que solo se asoció negativamente con el miedo a la devaluación de uno mismo y a perturbar a otros importantes. Por lo tanto, sería conveniente que el profesor fuera capaz de proponer tareas en los que los alumnos tuvieran mayores probabilidades de éxito, ya que en caso contrario, se sentirían incompetentes y comenzarían a desmotivarse (Deci y Ryan, 2000), incrementándose de este modo el miedo a tener un futuro incierto, lo que significaría que sentirían presión y agobio por las consecuencias que podría llegar a tener en su vida el cometer errores, así como el sentirse insignificantes para sus compañeros y profesores. Asimismo, y según la literatura, el profesor puede generar el sentimiento de competencia, mediante el fomento de un correcto clima motivacional, ya que esto 
influirá sobre la orientación disposicional del alumno en las clases de EF (Nicholls, 1989), favoreciendo así su capacidad de percepción de competencia.

Estos resultados coinciden parcialmente con estudios como los realizados por Silveira y Moreno (2015) y Smith et al. (2002) en los cuales la percepción de competencia además de coincidir en la relación significativa y negativa con las causas aversivas del miedo al fallo, también se asoció negativamente con el miedo a experimentar vergüenza, lo que puede deberse a que la muestra de este estudio es similar, ya que está compuesta por alumnos de EF de secundaria. Además, estudios realizados en el contexto deportivo con deportistas universitarios de edades superiores a los 18 años, también mostraron que el sentimiento de incompetencia se asoció con un mayor rechazo a la práctica de actividad física por lo que puedan pensar los demás ante un fallo (Zuckerman y Tsai, 2005).

Finalmente, resaltar que aquellos estudiantes que se relacionaron con el resto de los compañeros tuvieron menos miedo a experimentar vergüenza, devaluación de uno mismo, tener un futuro incierto, perder el interés de otros y perturbar a otros importantes. Por lo tanto, y a tenor de los resultados, el continuar fomentado las relaciones sociales de los alumnos con los profesores y con el resto de los compañeros es importante para evitar pensamientos negativos acerca de las opiniones o críticas que pueden recibir los estudiantes por parte del resto de compañeros ante los fallos, reduciendo de este modo el miedo a equivocarse en las clases de EF (Conroy, 2001; Sagar et al., 2007). Estos resultados coinciden con los hallados por Seifriz et al. (1992) en el ámbito deportivo con deportistas en edad escolar (14-19 años). Sin embargo, según el estudio de Silveira y Moreno (2015) el relacionarse con los demás solamente se relacionó de manera significativa y negativa con el miedo a experimentar vergüenza y a perder el interés de otros, mientras que no reflejó ninguna relación significativa con el resto de dimensiones del miedo al fallo, lo que puede deberse a que aunque la muestra estuvo formada por alumnos de EF, las edades fueron de 12 y 13 años, mientras que en este estudio se abarcó desde los 12 hasta los 17 años.

El presente estudio, además de aportar información novedosa sobre la relación entre la satisfacción de las NPB y la percepción del miedo el fallo, mostró algunas limitaciones que se deben mencionar. Entre estas limitaciones encontramos que los participantes son de centros educativos de la Región de Murcia lo que influye en la generalización de los resultados. Sin embargo, también hay que resaltar algunas fortalezas, como el tamaño de muestra analizada, la novedad de los resultados hallados, así como, la inclusión de una nueva variable como es el hábito de práctica físico-deportiva extraescolar.

A modo de conclusión, se demostró que la satisfacción de las NPB en las clases de EF de secundaria se relacionó negativamente con las conductas aversivas de miedo al fallo en el desarrollo de las actividades propuestas en clase. Teniendo en cuenta los resultados recogidos, el profesor debe proponer tareas que impliquen al alumno en el desarrollo de las tareas de clase, otorgándole importancia al proceso y al esfuerzo y restándole relevancia al resultado, para así conseguir altos niveles de satisfacción y de placer en la realización de las tareas, con el objetivo principal de conseguir que los alumnos realicen ejercicio físico de manera regular, llevando a cabo un estilo de vida activo y saludable. Para ello será fundamental, proponer más tareas donde los alumnos tomen sus propias decisiones y perciban mayor sensación de autonomía en las clases, ya que es esta la NPB que menos se satisface entre los alumnos, al tiempo que sigan sintiéndose competentes y continúen relacionándose con el resto de compañeros, ya que son las NPB que más satisfechas afirmaron tener los estudiantes, especialmente la relación con los demás, para así poder disminuir todas las 
SATISFACCIÓN DE LAS NECESIDADES PSICOLÓGICAS BÁSICAS EN LAS CLASES DE EDUCACIÓN FISICA Y SU RELACIÓN CON EL MIEDO AL FALLO SEGÚN EL GÉNERO Y LA PRÁCTICA FÍSICO-DEPORTIVA EXTRAESCOLAR ...

causas aversivas de miedo al fallo, sobre todo la del miedo a experimentar vergüenza y la devaluación de uno mismo que son las causas aversivas que más expresaron los estudiantes, entendiéndolo como un medio de aprendizaje, y no como un aspecto negativo.

Asimismo, es fundamental seguir fomentando la NPB de relación con los demás, ya que es la única que se relacionó negativa y significativamente con el miedo a experimentar vergüenza y a la devaluación de uno mismo. Por último, debe considerarse tanto el género como el hábito de práctica de ejercicio físico en su tiempo libre, ya que son dos factores que se ha demostrado que tienen relación con ambas variables de estudio.

Finalmente, sería necesario poder desarrollar un estudio con otras muestras pertenecientes a otras Comunidades Autónomas con el fin de generalizar los resultados. Además, también sería positivo poder relacionar las dos variables del estudio con otras como la satisfacción con la vida, estrategias motivacionales empleadas por el profesor de EF y el clima de aprendizaje y apoyo a las necesidades psicológicas básicas, para así poder profundizar más y conocer otros aspectos que puedan influir en los resultados. Para ello utilizaríamos otros instrumentos, como son los inventarios SWLS, CPEMEF y LCQ-EF.

\section{REFERENCIAS BIBLIOGRÁFICAS}

Aguado-Gómez, R., Díaz-Cueto, M., Hernández-Álvarez, J. L. \& López-Rodríguez, A. (2016). Apoyo a la autonomía en las clases de educación física: percepción versus realidad. Revista Internacional de Medicina y Ciencias de la Actividad Física y el Deporte, 16(62), 183-202. DOI: 10.15366/rimcafd2016.62.001

Amjad, R., Irshad, E. \& Gul, R. (2018). Relationship between competition anxiety and fear of failure among sportsmen and sportswomen. Journal of Postgraduate Medical Institute (PeshawarPakistan), 32(1), 9-65.

Amorose, A. J. \& Smith, P. (2003). Feedback as a source of physical competence information: Effects of age, experience and type of feedback. Journal of Sport and Exercise Psychology, 25(3), 341359. DOI: 10.1123 jsep.25.3.341

Baena-Extremera, A., Gómez-López, M., Granero-Gallegos, A. \& Martínez-Molina, M. (2016). Modelo de predicción de la satisfacción y diversión en Educación Física a partir de la autonomía y el clima motivacional. Universitas Psychologica, 15(2), 39-49. DOI: https://dx.doi. org/10.11144/Javeriana.upsy15-2.mpsd

Baena-Extremera, A., Gómez-López, M., Granero-Gallegos, A. \& Ortiz-Camacho, M. M. (2015). Predicting satisfaction in physical education from motivational climate and self-determined motivation. Journal of Teaching in Physical Education, 34(2), 210-224. DOI: 10.1123/jtpe.2013-0165

Balaguer, I., Castillo, I. \& Duda J. I. (2008). Apoyo a la autonomía, satisfacción de las necesidades, motivación y bienestar en deportistas de competición: Un análisis de la teoría de la autodeterminación. Revista de Psicología del Deporte, 17(1), 123-139.

Berglas, S. \& Jones, E. E. (1978) Drug choice as a self-handicapping strategy in response to noncontingent success. Journal of Personality and Social Psychology, 36(4), 405-417. DOI: http://dx.doi.org/10.1037/0022-3514.36.4.405

Black, A. E. \& Deci, E. L. (2000). The effects of instructors' autonomy support and students' autonomous motivation on learning organic chemistry: A self-determination theory perspective. Science Education, 84(6), 740-756.

Brunet, J. \& Sabiston, C. M. (2009). Social physique anxiety and physical activity: A selfdetermination theory perspective. Psychology of Sport and Exercise, 10(3), 329-335. DOI: 10.1016/j.psychsport.2008.11.002 
Estudios Pedagógicos XLVII N $1:$ 303-322, 2021

SATISFACCIÓN DE LAS NECESIDADES PSICOLÓGICAS BÁSICAS EN LAS CLASES DE EDUCACIÓN FÍSICA Y SU RELACIÓN CON EL MIEDO AL FALLO SEGÚN EL GÉNERO Y LA PRÁCTICA FÍSICO-DEPORTIVA EXTRAESCOLAR ...

Calvo, T. G., Miguel, P. A. S., Marcos, F. M. L., Oliva, D. S. \& Alonso, D. A. (2012). Análisis del grado de diversión e intención de persistencia en jóvenes deportistas desde la perspectiva de la teoría de la autodeterminación. Revista de Psicología del Deporte, 21(1), 7-13.

Cervelló, E. M., Jiménez, R., del Villar, F., Ramos, L. \& Santos-Rosa, F. J. (2004). Goal orientations, motivational climate, equality, and discipline of Spanish physical education students. Perceptual and Motor Skills, 99(1), 271-283. DOI: 10.2466/pms.99.1.271-283

Chatzisarantis, N. L. \& Hagger, M. S. (2009). Effects of an intervention based on self-determination theory on self-reported leisure-time physical activity participation. Psychology \& Health, 24(1), 29-48. DOI: 10.1080/08870440701809533

Chen, L. H., Chen, M. Y., Lin, M. S., Kee, Y. H. \& Shui, S. H. (2009). Fear of failure and selfhandicapping in college physical education. Psychological Reports, 105(3), 707-713. DOI: 10.2466/PR0.105.3.707-713

Chengfu, Y., Xian, L. \& Zhang, W. (2015). Predicting adolescent problematic online game use from teacher autonomy support, basic psychological needs satisfaction, and school engagement: A 2-Year Longitudinal Study. Cyberpsychology, Behavior and Social Networking, 18(4), 228-233

Conroy, D. E. (2001). Progress in the development of a multidimensional measure of fear of failure: The Performance Failure Appraisal Inventory. Anxiety, Stress \& Coping, 14(4), 431-452. DOI: $10.1080 / 10615800108248365$

Conroy, D. E. (2004). The unique psychological meanings of multidimensional fears of failing. Journal of Sport and Exercise Psychology, 26(3), 484-491. DOI: 10.1123/jsep.26.3.484

Conroy, D. E. \& Coastworth, J. D. (2007). Coaching behaviors associated with changes in fear of failure: Changes in self-talk and need satisfaction as potential mechanisms. Journal of Personality, 75(2), 383-419. DOI: 10.1111/j.1467-6494.2006.00443.x

Conroy, D. E., Poczwardowski, A. \& Henschen, K. P. (2001). Evaluative criteria and consequences associated with failure and success for elite athletes and performing artist. Journal of Applied Sport Psychology, 13(3), 300-322. DOI: 10.1080/104132001753144428

Conroy, D. E., Willow, J. P. \& Metzler, J. N. (2002). Multidimensional fear of failure measurement: The performance failure appraisal. Journal of Applied Sport Psychology, 14(2), 76-90. DOI: $10.1080 / 10413200252907752$

Correia, M., Rosado, A., Serpa, S. \& Ferreira, V. (2017). Fear of failure in athletes: gender, age and type of sport differences. Revista Iberoamericana de Psicología del Ejercicio y el Deporte, 12(2), 185-193

Cox, A. \& Williams L. (2008). The roles of perceived teacher support, motivational climate, and psychological need satisfaction in students' Physical Education motivation. Journal of Sport \& Exercise Psychology, 30(2), 222-239. DOI: 10.1123/jsep.30.2.222

De Castella, K., Byrne, D. \& Covington, M. (2013). Unmotivated or motivated to fail? A crosscultural study of achievement motivation, fear of failure, and student disengagement. Journal of Educational Psychology, 105(3), 861-880. DOI: https://doi.org/10.1037/a0032464

Deci, E. L. \& Ryan, R. M. (1985). Intrinsic motivation and self-determination in human behaviour. New York: Plenum.

. (1991). A motivational approach to self: Integration in personality. En R. A. Dienstbier (Ed.), Current theory and research in motivation, Vol. 38. Nebraska Symposium on Motivation, 1990: Perspectives on motivation (pp. 237-288). Lincoln, NE, US: University of Nebraska Press.

. (2000). The "what" and "why" of goal pursuits: Human needs and the self-determination of behaviour. Psychological Inquiry, 11(4), 227-268.

García, T., Sánchez, P. A., Leo, F. M., Sánchez, D. \& Amado, D. (2011). Incidencia de la Teoría de Autodeterminación sobre la persistencia deportiva. Revista Internacional de Ciencias del Deporte, 25(7), 266-276.

Gil, P., Campos, R. C., Jordán, O. R. C. \& Díaz, A. (2012). Educación Física y hábitos de vida activa: percepciones de los adolescentes y relación con el abandono deportivo. Aula Abierta, 40(3), $115-124$. 
Gómez, A., Hernández, J., Martínez, I. \& Gámez, S. (2014). Necesidades psicológicas básicas en Educación Física según el género y el ciclo educativo del estudiante durante la escolaridad obligatoria. Revista de Investigación Educativa, 32(1), 159-167. DOI: 10.6018/rie.32.1.172311

Gómez-López, M., Granero-Gallegos, A., Baena-Extremera, A. \& Abraldes, J. (2014). Análisis de los perfiles motivacionales y su relación con la importancia de la educación física en secundaria. Revista Iberoamericana de Diagnóstico y Evaluación Psicológica, 2(38), 11-29

Gómez-López, M., Granero-Gallegos, A., Baena-Extremera, A., Bracho, C. \& Pérez, F. J. (2015). Efectos de interacción de sexo y práctica de ejercicio físico sobre las estrategias para la disciplina, motivación y satisfacción con la Educación Física. Revista Iberoamericana de Diagnóstico y Evaluación, 1(40), 6-16.

Goudas, M., Biddle, S. \& Fox, K. (1994). Perceived locus of causality, goal orientations, and perceived competence in school physical education classes. British Journal of Educational Psychology, 64(3), 453-463. DOI: 10.1111/j.2044-8279.1994.tb01116.x

Goulimaris, D., Mavridis, G., Genti, M. \& Rokka, S. (2014). Relationships between basic psychological needs and psychological well-being in recreational dance activities. Journal of Physical Education and Sport, 14(2), 277-284.

Granero-Gallegos, A. \& Baena-Extremera, A. (2014). Predicción de la motivación autodeterminada según las orientaciones de meta y el clima motivacional en Educación Física. Retos. Nuevas tendencias en Educación Física, Deporte y Recreación, 25, 23-27.

Gunnell, K. E., Crocker, P. R., Wilson, P. M., Mack, D. E. \& Zumbo, B. D. (2013). Psychological need satisfaction and thwarting: A test of basic psychological needs theory in physical activity contexts. Psychology of Sport and Exercise, 14(5), 599-607. DOI: 10.1016/j.psychsport.2013.03.007

Gutiérrez, D., Garcia-Lopez, L. M., Hastie, P. A. \& Calderon, A. (2013). Spanish students' perceptions of their participation in seasons of sport education. The Global Journal of Health and Physical Education Pedagogy, 2(2), 111-127.

Harris, K. C., Kuramoto, L. K., Schulzer, M. \& Retallack, J. E. (2009). Effect of school-based physical activity interventions on body mass index in children: a meta-analysis. Canadian Medical Association Journal, 180(7), 719-726. DOI: 10.1503/cmaj.080966

Heaven, P. C. L. (1996). Adolescent's health: The role of individual differences. Londres: Routledge.

Leptokaridou, E. T., Vlachopoulos, S. P. \& Papaioannou, A. G. (2015). Associations of autonomy, competence, and relatedness with enjoyment and effort in elementary school physical education: The mediating role of self-determined motivation. Hellenic Journal of Psychology, 12, 105-128.

Leversen, I., Danielsen, A. G., Birkeland, M. S. \& Samdal, O. (2012). Basic psychological need satisfaction in leisure activities and adolescents' life satisfaction. Journal of Youth and Adolescence, 41(12), 1588-1599. DOI: https://doi.org/10.1007/s10964-012-9776-5

Lim, B. C. \& Wang, C. J. (2009). Perceived autonomy support, behavioural regulations in physical education and physical activity intention. Psychology of Sport and Exercise, 10(1), 52-60. DOI: 10.1016/j.psychsport.2008.06.003

McGregor, H. A. \& Elliot, A. J. (2005). The shame of failure: Examining the link between fear of failure and shame. Personality and Social Psychology Bulletin, 31(2), 218-231. DOI: $10.1177 / 0146167204271420$

Méndez-Giménez, A., Fernández-Río, J. \& Cecchini Estrada, J. A. (2013). Papel importante del alumnado, necesidades psicológicas básicas, regulaciones motivacionales y autoconcepto físico en educación física. Cuadernos de Psicología del Deporte, 13(1), 71-82.

Menéndez Santurio, J. I. \& Fernández-Río, J. (2018). Versión española de la escala de necesidades psicológicas básicas en educación física. Revista Internacional de Medicina y Ciencias de la Actividad Física y del Deporte, 18(69), 119-133. DOI: 10.15366/rimcafd2018.69.008

Monteiro, D., Machado, S., Moutão, J., Bento, T., Vitorino, A. \& Cid, L. (2019). Ejercicio físico y estilo de vida sedentario: consecuencias para la salud. Espiral. Cuadernos del Profesorado, 12(25), 75-88. DOI: http://dx.doi.org/10.25115/ecp.v12i25 
Estudios Pedagógicos XLVII N 1: 303-322, 2021

SATISFACCIÓN DE LAS NECESIDADES PSICOLÓGICAS BÁSICAS EN LAS CLASES DE EDUCACIÓN FÍSICA Y SU RELACIÓN CON EL MIEDO AL FALLO SEGÚN EL GÉNERO Y LA PRÁCTICA FÍSICO-DEPORTIVA EXTRAESCOLAR ...

Moreno, B., Jiménez, R., Gil, A., Aspano, M. I. \& Torrero, F. (2011). Análisis de la percepción del clima motivacional, necesidades psicológicas básicas, motivación autodeterminada y conductas de disciplina de estudiantes adolescentes en las clases de educación física. Motricidad. European Journal of Human Movement, 26, 1-24.

Moreno, J. A., Cervelló, E., Montero, C., Vera, J. A. \& García, T. (2012). Metas sociales, necesidades psicológicas básicas y motivación intrínseca como predictores de la percepción del esfuerzo en las clases de educación física. Revista de Psicología del Deporte, 21(2), 215-221.

Moreno, J. A., Zomeño, T. E., Marín, L. M., Ruiz, L. M. \& Cervelló, E. (2013). Percepción de la utilidad e importancia de la educación física según la motivación generada por el docente. Revista de Educación, 362, 380-402.

Moreno-Murcia, J. A, González-Cutre, D., Chillón, M. \& Parra, N. (2008). Adaptación a la Educación Física de la Escala de las Necesidades Psicológicas Básicas en el ejercicio. Revista Mexicana de Psicología, 25(2), 295-303.

Moreno-Murcia, J. A. \& Conte, L. (2011). Predicción del miedo a equivocarse en jugadores de baloncesto a través del clima tarea de los iguales y la motivación intrínseca. Revista Mexicana de Psicología, 28(1), 43-52.

Moreno-Murcia, J. A., Conte, L., Silveira, Y. \& Ruíz, L. M. (2014). Miedo a fallar en el deporte. Elche: Universidad Miguel Hernández de Elche.

Moreno-Murcia, J. A., Ferriz, R., Belando, N. \& Conte, L. (2011). Miedo a equivocarse en jóvenes jugadores de baloncesto: Diferencias por sexo. En Actas del II Congreso del Deporte en Edad Escolar. Universidad Miguel Hernández de Elche y Universidad de Murcia.

Moreno-Murcia, J. A., Martínez-Galindo, C., Belando, N. \& Conte, L. (2010). Relación del clima tarea de los iguales con el miedo a equivocarse en el deporte. Diferencias por sexo en una muestra de jugadores de baloncesto. En Actas del I Congreso Internacional de Cultura y Género: La cultura del culto al cuerpo. Elche: Universidad Miguel Hernández de Elche.

Moreno-Murcia, J. A., Silveira, Y. \& Conte, L. (2013). Relación del feed-back positivo y el miedo a fallar sobre la motivación intrínseca. Revista Española de Orientación y Psicopedagogía, 24(2), 8-23. DOI: 10.5944/reop.vol.24.num.2.2013.11256

Munuera, J. M., Gómez-López, M., Granero-Gallegos, A. \& Sánchez-Alcaraz, B. J. (2018). Miedo al fallo de los estudiantes y la relación con la intención de ser físicamente activo e importancia de la Educación Física en Educación Secundaria. Revista Estudios Pedagógicos, XLIV(1), 279-291.

Nicaise, V., Cogerino, G., Bois, J. \& Amorose, A. J. (2006). Students' perceptions of teacher feedback and physical competence in physical education classes: Gender effects. Journal of Teaching in Physical Education, 25(1), 36-57.

Nicholls, J. G. (1989). The competitive ethos and democratic education. Cambridge, MA: Harvard University Press.

Ntoumanis, N. (2001). A self-determination approach to the understanding of motivation in physical education. British Journal of Educational Psychology, 71(2), 225 242. DOI: 10.1348/000709901158497

Orkibi, H. \& Ronen, T. (2017). Basic Psychological Needs Satisfaction Mediates the Association between Self-Control Skills and Subjective Well-Being. Frontiers in psychology, 8(936), 1-10. DOI: $10.3389 /$ fpsyg.2017.00936

Pensgaard, A. M. \& Roberts, G. C. (2000). The relationship between motivational climate, perceived ability and sources of distress among elite athletes. Journal of Sports Sciences, 18(3), 191-200. DOI: $10.1080 / 026404100365090$

Ruiz-Sánchez, V., Gómez-López, M., Granero-Gallegos, A. \& González, J. (2017). Relación del clima motivacional y miedo al fallo en jugadores de alto rendimiento en Balonmano. Cuadernos de Psicología del Deporte, 17(3), 55-64.

Ryan, R. M. \& Deci, E. L. (2000). Self-determination theory and the facilitation of intrinsic motivation, social development and well-being. American Psychologist, 55(1), 68-78. DOI: 10.1037/0003-066X.55.1.68 
SATISFACCIÓN DE LAS NECESIDADES PSICOLÓGICAS BÁSICAS EN LAS CLASES DE EDUCACIÓN FÍSICA Y SU RELACIÓN CON EL MIEDO AL FALLO SEGÚN EL GÉNERO Y LA PRÁCTICA FÍSICO-DEPORTIVA EXTRAESCOLAR ...

Ryan, R. M., Kuhl, J. \& Deci, E. L. (1997). Nature and autonomy: Organizational view of social and neurobiological aspects of self-regulation in behavior and development. Development and Psychopathology, 9(4), 701-728. DOI: 10.1017/S0954579497001405

Sagar, S. S. \& Stoeber, J. (2009). Perfectionism, fear of failure and affective responses to success and failure: The central role of fear of experiencing shame and embarrassment. Journal of Sport and Exercise Psychology, 31(5), 602-627. DOI: 10.1123/jsep.31.5.602

Sagar, S. S., Busch, B. \& Jowett, S., (2010). Success and failure, fear of failure, and coping responses of adolescent Academy Football Players. Journal of Applied Sport Psychology, 22(2), 213-230. DOI: $10.1080 / 10413201003664962$

Sagar, S. S., Lavallee, D. \& Spray, C. M. (2007). Why young elite athletes fear failure: Consequences of failure. Journal of Sports Sciences, 25(11), 1171-1184. DOI: 10.1080/02640410601040093

Sagar, S., Boardley, I. \& Kavussanu, M. (2010). Fear of failure and student athletes' interpersonal antisocial behaviour in education and sport. British Journal of Educational Psychology, 81(3), 391-408. DOI: 10.1348/2044-8279.002001

Sánchez-Alcaraz, B. J., Murcia Gálvez, S., Alfonso-Asencio, M. \& Hellín-Martínez, M. (2020). Nivel de actividad física en estudiantes en función de la edad, el género, tipo de deporte practicado y su orientación motivacional. Espiral. Cuadernos del Profesorado, 13(27), 160-169. DOI: http:// dx.doi.org/10.25115/ecp.v13i27

Seifriz, J., Duda, J. L. \& Chi, L. (1992). The relationship of perceived motivational climate to intrinsic motivation and beliefs about success in basketball. Journal of Sport and Exercise Psychology, 14(4), 375-391.

Sheldon, K. M. \& Elliot, A. J. (1999). Goal striving, need satisfaction and longitudinal well-being: The Self-Concordance Model. Journal of Personality and Social Psychology, 76(3), 482-497. DOI: $10.1037 / 0022-3514.76 .3 .482$

Silveira, Y. \& Moreno, J. A. (2015). Miedo a equivocarse y motivación autodeterminada en estudiantes adolescentes. Cuadernos de Psicología del Deporte, 15(3), 65-74.

Smith, L., Sinclair, K. E. \& Chapman, E. S. (2002). Students' goals, self-efficacy, self-handicapping, and negative affective responses: An Australian senior school student study. Contemporary Educational Psychology, 27(3), 471-485. DOI: 10.1006/ceps.2001.1105

Standage, M., Duda, J. L. \& Ntoumanis, N. (2003). A model of contextual motivation in physical education: Using constructs from self-determination and achievement goal theories to predict physical activity intentions. Journal of Educational Psychology, 95(1), 97-110. DOI: 10.1037/0022-0663.95.1.97

. (2005). A test of self-determination theory in school physical education. British Journal of Educational Psychology, 75(3), 411-433. DOI: 10.1348/000709904X22359

Taylor, I. M., Ntoumanis, N., Standage, M. \& Spray, C. M. (2010). Motivational predictors of physical education students' effort, exercise intentions, and leisure-time physical activity: A multilevel linear growth analysis. Journal of Sport and Exercise Psychology, 32(1), 99-120.

Timo, J., Sami, Y. P., Anthony, W. \& Jarmo, L. (2016). Perceived physical competence towards physical activity, and motivation and enjoyment in physical education as longitudinal predictors of adolescents' self-reported physical activity. Journal of Science and Medicine in Sport, 19(9), 750-754. DOI: 10.1016/j.jsams.2015.11.003

Vilhjalmsson, R. \& Thorlindsson, T. (1998). Factors related to physical activity: a study of adolescents. Social Science and Medicine, 47(5), 665-675.

Vlachopoulos, S. P. \& Michailidou, S. (2006). Development and initial validation of a measure of autonomy, competence, and relatedness: the Basic Psychological Needs in Exercise Scale. Measurement in Physical Education and Exercise Science, 10(3), 179-201. DOI: 10.1207/s15327841mpee1003_4

Vlachopoulos, S. P., Katartzi, E. S. \& Kontou, M. G. (2011). The Basic Psychological Needs in Physical Education Scale. Journal of Teaching in Physical Education, 30(3), 263-280. DOI: 10.1123/jtpe.30.3.263 
Estudios Pedagógicos XLVII N ${ }^{\circ}$ : 303-322, 2021

SATISFACCIÓN DE LAS NECESIDADES PSICOLÓGICAS BÁSICAS EN LAS CLASES DE EDUCACIÓN FÍSICA Y SU

RELACIÓN CON EL MIEDO AL FALLO SEGÚN EL GÉNERO Y LA PRÁCTICA FÍSICO-DEPORTIVA EXTRAESCOLAR ...

Xiang, P., Agbuga, B., Liu, J. \& McBride, R. E. (2017). Relatedness need satisfaction, intrinsic motivation, and engagement in secondary school physical education. Journal of Teaching in Physical Education, 36(3), 340-352. DOI: 10.1123/jtpe.2017-0034

Zhang, T., Solmon, M. A. \& Gu, X. (2012). The role of teachers' support in predicting students' motivation and achievement outcomes in physical education. Journal of Teaching in Physical Education, 31(4), 329-343.

Zuckerman, M. \& Tsai, F. (2005). Costs of self-handicapping. Journal of Personality, 73(2), 411-442. DOI: 10.1111/j.1467-6494.2005.00314.x 